\title{
Heisenberg (and Schrödinger, and Pauli) on Hidden Variables
}

\author{
Guido Bacciagaluppi*and Elise Crull ${ }^{\dagger}$
}

10 July 2009

\begin{abstract}
In this paper, we discuss various aspects of Heisenberg's thought on hidden variables in the period 1927-1935. We also compare Heisenberg's approach to others current at the time, specifically that embodied by von Neumann's impossibility proof, but also views expressed mainly in correspondence by Pauli and by Schrödinger. We shall base ourselves mostly on published and unpublished materials that are known but littlestudied, among others Heisenberg's own draft response to the EPR paper. Our aim will be not only to clarify Heisenberg's thought on the hidden-variables question, but in part also to clarify how this question was understood more generally at the time.
\end{abstract}

\section{Introduction}

In modern discussions, one normally distinguishes between two notions of hidden variables: non-contextual and contextual ones. Non-contextual hidden variables are those that fix values or probabilities or expectation values for all quantum mechanical observables, independent of any experimental context. The impossibility proofs of von Neumann (1932), Gleason (1957), and Kochen and Specker (1967) refer to this kind of hidden variables.

In his celebrated paper of 1966, J. S. Bell criticised these classic impossibility proofs, pointing out that what is actually required of a hidden-variables theory is merely that it explain how a definite result is produced in each measurement, in a way that may depend indeed on the context of the experiment, as is the case in Bohm's 1952 theory. Since Bohm's theory is not only contextual but non-locally so, Bell was led to investigate locality constraints on hidden variables theories, proving his famous inequalities (Bell 1964) and subsequently generalising them to the case of stochastic hidden variables theories.

As we shall see, elements of the modern conception of hidden variables were already present in the time period we are concerned with (1927-1935). However, the discussion

\footnotetext{
${ }^{*}$ Department of Philosophy, University of Aberdeen, The Old Brewery, High Street, Aberdeen AB24 3UB, U. K.; email: g.bacciagaluppi@abdn.ac.uk .

${ }^{\dagger}$ Program in History and Philosophy of Science, Department of Philosophy, University of Notre Dame, Notre Dame, IN 46556, U. S. A.; email: ecrull@nd.edu .
} 
in this period was often in terms of whether quantum mechanical particles admitted of a more complete description, in which both positions and momenta (and other classical variables) had definite values, which furthermore evolved deterministically. We shall use the term 'hidden variables' as a blanket to denote both the current understanding of hidden variables theories and earlier discussions. Our aim will be to clarify at least in part how these questions were understood in this earlier period.

We first discuss various aspects of Heisenberg's thought on hidden variables up to 1935: in particular, the tension Heisenberg sees between hidden variables and interference effects, and the role of the movability of the cut between observer and observed. We then compare Heisenberg's approach to others current at the time, specifically that embodied by von Neumann's impossibility proof, but also views expressed mainly in correspondence by Pauli and especially Schrödinger. We shall base ourselves mostly on published and unpublished materials that are known but little-studied, among others Heisenberg's own draft response to the paper by Einstein, Podolsky and Rosen (1935, henceforth EPR). ${ }^{1}$

\section{Heisenberg's early views}

In this section, we discuss two aspects of Heisenberg's views that we consider central to his thought on hidden variables, namely: his views on indeterminism as set out in particular in correspondence with Einstein in 1927, and his views on interference as set out in particular in his joint report with Born at the 1927 Solvay conference. For more details on both (especially the latter), see Bacciagaluppi (2008) and Bacciagaluppi and Valentini (2009).

\subsection{The 1927 Heisenberg-Einstein correspondence}

In May 1927, Einstein had proposed what in retrospect appears to be an alternative version of pilot-wave theory. This theory was described in a talk to the Prussian Academy of Sciences ${ }^{2}$ but Einstein withdrew the paper from publication. Heisenberg had heard of the paper and wrote to Einstein about it (it is unlikely that he ever saw a copy, though). Of this correspondence, two letters survive, both from Heisenberg to Einstein, ${ }^{3}$ and the second of these letters spells out precisely Heisenberg's arguments against hidden variables, or more precisely why Heisenberg believes indeterminism is 'necessary, not just consistently possible'.

\footnotetext{
${ }^{1}$ All original translations are ours.

${ }^{2}$ 'Bestimmt Schrödingers Wellenmechanik die Bewegung des Systems vollständig oder nur im Sinne der Statistik?', Albert Einstein Archive 2-100.00 (in German); currently available on-line at http://www.alberteinstein.info/db/ViewDetails.do?DocumentID=34338. See also Belousek (1996) and Holland (2005).

${ }^{3}$ Heisenberg to Einstein, 19 May and 10 June 1927, Albert Einstein Archive 12-173.00 and 12-174.00 (both in German).
} 
Heisenberg's argument is based on the consideration of electron diffraction. Heisenberg imagines free electrons impinging on a grating with spacing comparable to their de Broglie wavelength. If these electrons indeed have trajectories and if they behave like particles, i.e. interact via short-range interactions, ${ }^{4}$ then it would be possible for a small obstacle placed at some point along the grating to deflect the electron in an arbitrary direction. However, the electron is deflected only in the discrete directions that depend on the global properties of the grating, thereby giving rise to the observed interference pattern. As Heisenberg notes, the only way to have the direction of scattering depend on the features of the grating other than where the particle hits it, is to make the trajectory of the particle depend on the associated wave rather than on particle-like short-range interactions. But in Heisenberg's opinion that would amount to giving up the notion of a 'particle'.

The essence of the argument as we see it is that the existence of hidden variables (here in the sense of deterministic trajectories) will suppress interference effects, and is thus incompatible with the predictions of quantum mechanics. We shall see this core idea repeatedly in what follows.

\subsection{Born and Heisenberg's Solvay report}

The 1927 Solvay conference was the fifth in the celebrated series of conferences funded by the Belgian industrialist and philanthropist Ernest Solvay and organised for the most part through the untiring energy of H. A. Lorentz. The 1927 conference was particularly important in the discussions about the foundational status of quantum mechanics. In particular, de Broglie presented his pilot-wave theory in detail, but failed to convince his 'mainstream' colleagues of its merits, Schrödinger and (jointly) Born and Heisenberg presented their still quite opposing views on wave mechanics and quantum mechanics, respectively, and in the sidelines Bohr and Einstein engaged in informal discussions that have become famous in their own right. ${ }^{5}$

Born and Heisenberg's (1928) report contains arguably the most detailed presentation of the statistical interpretation of quantum mechanics. Until this report, the most explicit presentation of the statistical interpretation of quantum theory was that given in Born's paper on the adiabatic theorem (1926), which lacks, however, a separate discussion of interference; and this is the crucial point where the report by Born and Heisenberg goes further, with a treatment of interference that is quite remarkable.

\footnotetext{
${ }^{4}$ As suggested by the anonymous referee, this can be understood as a kind of locality requirement. Note, however, that while locality considerations in the case of two entangled particles are very powerful (as shown by Bell's theorem), in the case of one particle these considerations are a double-edged sword. Indeed, Einstein at the fifth Solvay conference (October 1927) used locality considerations in a one-particle, single-slit diffraction setup to argue for the existence of trajectories (Bacciagaluppi and Valentini 2009, pp. 486-488).

${ }^{5} \mathrm{~A}$ translation of the proceedings of the conference and an extended analysis of their significance is given in Bacciagaluppi and Valentini (2009). Page references are to the on-line draft of this translation. The Bohr-Einstein exchange is described in Bohr (1949).
} 
Born and Heisenberg (p. 423) consider an atom that is initially in a superposition of energy states $\psi_{n}(x)$, with coefficients $c_{n}(0)=\left|c_{n}(0)\right| e^{i \gamma_{n}}$ and eigenvalues $E_{n}$. Under the Schrödinger equation the coefficients evolve as

$$
c_{n}(t)=\sum_{m} S_{n m}(t) c_{m}(0)
$$

As in Born's paper, Born and Heisenberg point out that, in the special case where $c_{m}(0)=$ $\delta_{m k}$ for some $k$, we have $\left|c_{n}(t)\right|^{2}=\left|S_{n k}(t)\right|^{2}$, and one can interpret $\left|S_{n k}(t)\right|^{2}$ as a transition probability. Consequently, 'the $\left|c_{n}(t)\right|^{2}$ must be the state probabilities' (p. 424).

At this point, however, Born and Heisenberg recognise a 'difficulty of principle' (p. 424), which is precisely that for an initial superposition of energy states the final probability distribution is given by

$$
\left|c_{n}(t)\right|^{2}=\left|\sum_{m} S_{n m}(t) c_{m}(0)\right|^{2}
$$

as opposed to

$$
\left|c_{n}(t)\right|^{2}=\sum_{m}\left|S_{n m}(t)\right|^{2}\left|c_{m}(0)\right|^{2}
$$

This 'theorem of the interference of probabilities' in Born and Heisenberg's words appears to contradict what 'one might suppose from the usual probability calculus' (p. 424).

Born and Heisenberg then make a remarkable statement (pp. 424-425):

[...] it should be noted that this 'interference' does not represent a contradiction with the rules of the probability calculus, that is, with the assumption that the $\left|S_{n k}\right|^{2}$ are quite usual probabilities. In fact, [...] [(3)] follows from the concept of probability [...] when and only when the relative number, that is, the probability $\left|c_{n}\right|^{2}$ of the atoms in the state $n$, has been established beforehand experimentally. In this case the phases $\gamma_{n}$ are unknown in principle, so that $[(2)]$ then naturally goes over to $[(3)][\ldots]$.

What Born and Heisenberg say here is that the expressions $\left|S_{n k}\right|^{2}$ denote 'usual' transition probabilities irrespectively of whether they appear in (2) or in (3). Instead, the reason for the failure of (3) to hold in general is that the expressions $\left|c_{m}\right|^{2}$ are not always state probabilities, because the state probabilities themselves are not always well-defined (cf. Bacciagaluppi and Valentini 2009, pp. 175-176). If the state probabilities are well-defined (namely if the energy has been measured, in general non-selectively), then one can calculate them at future times using (3). The truth of this conditional statement, however, is not affected if the state probabilities are not well-defined.

A few remarks are in order. First of all, this treatment of the law of total probability is analogous to Heisenberg's famous discussion of the 'law of causality' in his uncertainty paper: the law is again a conditional statement, which remains true although the state of the system is defined in fact only to within the accuracy given by the uncertainty 
principle. In Heisenberg's own words: '[...] in the sharp formulation of the law of causality, "If we know the present exactly, we can calculate the future", it is not the consequent that is wrong, but the antecedent. We cannot in principle get to know the present in all determining data' (Heisenberg 1927, p. 197). ${ }^{6}$

Second, note that what Born and Heisenberg mean by 'usual' transition probabilities bears some resemblance to the notion of potentialities or propensities familiar from the philosophy of probability starting in the 1950s (e.g. Popper 1959). Indeed, Born and Heisenberg's transition probabilities are well-defined even when there is no value could make a transition. They are like some probabilistic 'field of force', existing independently of the presence of a 'test particle'.

Third, note also that in this discussion (as well as in Heisenberg's uncertainty paper) stationary states no longer have a privileged status per se, but only acquire it through the experimental context.

Finally, and crucially for our purposes, we see again the idea that the existence of hidden values would destroy interference, but the mechanism for this is now made more explicit than in Heisenberg's 1927 letter to Einstein. Indeed, since the transition probabilities $\left|S_{n k}\right|^{2}$ are 'usual' probabilities, we necessarily have that if (a distribution of) values for the energy were well-defined, the interference formula (2) would cease to hold, and would go over to (3). Born and Heisenberg explain interference precisely through the absence of any 'hidden' values for the energy.

We now move on to a further stage of development of Heisenberg's ideas.

\section{Heisenberg's 1935 response to EPR}

Immediately following the publication of the EPR paper in the spring of 1935, Pauli wrote to Heisenberg and suggested that Heisenberg develop his own response to EPR - despite the fact that Bohr was already working on a response. The implication was that Heisenberg, as Pauli well knew, would provide a rather different argument in defense of quantum mechanics (or against the possibility of ever rendering it 'complete', as Einstein, Podolsky and Rosen desired) than the strategy Bohr would likely employ.

Heisenberg took up Pauli's suggestion. On 2 July, he wrote to Pauli: 'Since [Bohr's] reply is very different from the thoughts that one might express in connection with the "cut", I am actually still eager to write something about the cut in line with your suggestion' (Pauli 1985, p. 408). And that is precisely what he does, enclosing with the letter to Pauli a draft of his response to EPR titled: 'Ist eine deterministische Ergänzung der Quantenmechanik möglich?' This paper would never be published, save for a transcription, in German, in the multi-volume collection of Pauli's scientific correspondence (Pauli 1985,

\footnotetext{
${ }^{6}$ On Heisenberg's treatment of the 'law of causality', see also Beller (1999), pp. 110-113.

${ }^{7}$ Note that in later years, Heisenberg expressed explicitly a view of quantum probabilities in terms of propensities (Heisenberg 1958, esp. Chapter III).
} 
pp. $409-418){ }^{8}$

As promised, Heisenberg's draft takes the cut argument as central. Although Heisenberg had spoken of the 'cut' several times in the years prior to 1935 and would continue to use this specific argument in writings and lectures afterward, it is within his response to the EPR paper that we find the most carefully laid-out and detailed instance of it. The general idea is to demonstrate the 'incompletability' of quantum mechanics through the necessary conceptual divide between the observer and the observed - this divide being referred to as the 'Schnitt', or cut, between those parts of a system to be treated classically, and those to be treated quantum mechanically. Furthermore, it is within the wider context of Heisenberg's cut argument (as presented in his 1935) that his fullest argument against the possibility of hidden variables is situated.

Briefly, the structure of the 1935 draft is as follows: after a few introductory remarks reminding the reader of the assumptions of quantum mechanics and its basic formalism, Heisenberg introduces the cut argument, whose two main components we discuss below. After the cut argument is explained, Heisenberg segues to a consideration of the possibility of hidden variables, and ends the essay with a discussion of observational context.

Although we shall not focus on Heisenberg's remarks regarding the latter topic, it is worth noting that in addressing it, Heisenberg states that the statistical element in quantum mechanics is a necessary consequence of the discontinuity arising from the switch from one observational context to another "in the Bohrian sense "complementary"' one.

\subsection{The cut argument}

As alluded to above, Heisenberg developed versions of his cut argument in various places prior to and after 1935. In the Chicago lectures (delivered in the spring of 1929), Heisenberg presents aspects of the cut argument in conjunction with a discussion of interference that parallels his discussion with Born in the 1927 Solvay report. ${ }^{9}$ In 1934 Heisenberg gave a lecture in Hannover for a gathering of the Gesellschaft deutscher Naturforscher und Ärzte titled 'Wandlungen in den Grundlagen der exakten Naturwissenschaft in jüngster Zeit' (Heisenberg 1949, pp. 7-21). Within this lecture, Heisenberg mentions the impossibility of completing quantum mechanics based on considerations relating to the cut. While he does present many of the claims that would later become central to his cut argument as presented in his (1935), he does little in the way of arguing for these claims. Likewise, in a lecture given by Heisenberg at the University of Vienna in November of 1935, 'Prinzipielle Fragen der modernen Physik' (Heisenberg 1949, pp. 35-46), he mentions again the basic elements of the cut argument, and adds a presentation of the thought experiment (involving

\footnotetext{
${ }^{8}$ We are currently preparing a translation of this manuscript, as well as correcting various transcription errors. Heisenberg's manuscript can be viewed in the Archive for the History of Quantum Physics, microfilm 45, section 11 (in German).

${ }^{9} \mathrm{Cf}$. chapter 4 in his (1930). Although in this treatment Heisenberg evidently thinks these two issues are connected, he does not make the connection explicit.
} 
the refraction of an $\alpha$-particle) he had described in his 1927 letter to Einstein. As we shall see, Heisenberg's 1927 argument for the impossibility of hidden variables functions as part of his wider arguments involving the cut. Before getting to the question of hidden variables, then, let us first see how Heisenberg presents the cut argument in his 1935 draft.

Discussion of the cut contains two main components: the claim that the placement of the cut is arbitrary, and the claim that the statistical element can only appear at the place of the cut. Each claim is argued for separately by Heisenberg in his 1935.

Heisenberg argues for the first claim (arbitrary placement of the cut) by imagining the different components of an experimental apparatus: the atomic system $A$ to be measured, a chain of measuring devices $B, C$, etc., and the observer. He then claims that the probability for registering a specific reaction in the process of measurement is independent of the placement of the cut, except for considerations related to the sensitivity of the measuring devices.

Three different scenarios are considered to demonstrate this point: a case in which (i) the cut is located between atomic system $A$ and the first measuring device $B$, (ii) the cut is located beyond $B$ yet before $C$, in which case $C$ is considered as the measuring device, and (iii) the cut is located at any other point beyond device $C$ (yet not beyond the observer).

In case (i), the atomic system $A$ is of course treated quantum mechanically while measuring device $B$ is treated classically. Heisenberg thus assigns the time-dependent wavefunction $\psi_{A}\left(q_{A}, t\right)$ to the processes of system $A$. It follows that the probability that the coordinates $q_{A}$ of system $A$ will assume the particular value $q_{A}^{\prime}$ at the time of interaction between the atomic system and the measuring device $t^{\prime}$ is given by $\left|\psi_{A}\left(q_{A}^{\prime}, t^{\prime}\right)\right|^{2}$.

In case (ii), the atomic system and the device $B$ are treated quantum mechanically as the composite system $A+B$, and the other side of the cut contains the new measuring device $C$, again treated classically. Here Heisenberg must demonstrate that the probability of $B$ showing the specific value $q_{A}^{\prime}$ upon interaction with the system $A$ corresponds to the probability given in case (i). Prior to any interaction between $A$ and $B$, the wavefuction of the ensemble is given by the product of the undisturbed individual wavefunctions: $\psi_{A}\left(q_{A}, t\right) \cdot \psi_{B}\left(q_{B}, t\right)$. Now, write $H_{A}$ for the Hamiltonian of system $A, H_{B}$ for that of system $B$, and $H_{A B}$ for the interaction Hamiltonian. Schrödinger's equation for this system is then given as follows:

$$
\left(\frac{\hbar}{i} \frac{d}{d t}+H_{A}+H_{B}\right) \psi\left(q_{A}, q_{B}, t\right)=-H_{A B} \psi\left(q_{A}, q_{B}, t\right)
$$

As Heisenberg states, at $t=t^{\prime}$ the state $\psi\left(q_{A}, q_{B}, t\right)$ is given by the product of the undisturbed wavefunctions, $\psi_{A}\left(q_{A}, t^{\prime}\right) \cdot \psi_{B}\left(q_{B}, t^{\prime}\right)$. Furthermore, since $H_{A B}$ is zero other than for $q_{A}=q_{A}^{\prime}$, the right-hand side of (4) becomes $-H_{A B} \psi_{A}\left(q_{A}^{\prime}, t^{\prime}\right) \psi_{B}\left(q_{B}, t^{\prime}\right)$. Heisenberg then states without proof that the solution to the Schrödinger equation has the form

$$
\psi\left(q_{A}, q_{B}, t\right)=\psi_{A}\left(q_{A}, t\right) \psi_{B}\left(q_{B}, t\right)+\psi_{A}\left(q_{A}^{\prime}, t^{\prime}\right) \varphi\left(q_{A}, q_{B}, t, t^{\prime}\right)
$$


where $\varphi\left(q_{A}, q_{B}, t, t^{\prime}\right)$ is independent of the state of system $A$ before $t=t^{\prime}$.

Heisenberg then claims that the probability for $B$ to obtain the particular result $q_{A}^{\prime}$ in the measurement of the coordinate $q_{A}$ of system $A$ at time $t^{\prime}$ is given by

$$
\int d q_{A} d q_{B}\left|\psi_{A}\left(q_{A}^{\prime}, t^{\prime}\right) \varphi\left(q_{A}, q_{B}, t, t^{\prime}\right)\right|^{2}=\left|\psi_{A}\left(q_{A}^{\prime}, t^{\prime}\right)\right|^{2} \int d q_{A} d q_{B}\left|\varphi\left(q_{A}, q_{B}, t, t^{\prime}\right)\right|^{2},
$$

which is proportional to the probability outcome of case (i).

In case (iii), where the cut is removed beyond any number of measuring devices, the calculation is essentially the same as in case (ii). Given these three cases, which exhaust all possible locations of the cut, Heisenberg is able to conclude that the predictions of quantum mechanics regarding a specific measurement outcome are the same irrespective of the placement of the cut (up to a factor depending on the sensitivity of the measuring instruments).

An important qualification is mentioned here by Heisenberg: the placement of the cut is not completely arbitrary - for the measuring device $B$ could be a microscopic system that always needs to be considered on the quantum mechanical side of the cut. Thus, while the cut can be removed arbitrarily far in the direction of the observer, it cannot be removed arbitrarily far in the direction of the measured system.

There are problems with Heisenberg's proof of the arbitrariness of the placement of the cut. For instance, without also considering the sensitivity of the measurement in case (i), Heisenberg cannot show that the probabilities for registering the outcome are the same in both cases (i) and (ii). Furthermore, (6) is presumably meant as an instance of the standard Born rule, which assumes that the two terms in (5) are orthogonal. But that is inconsistent with the normalisation of the wavefunction, because the first term is already normalised. For our purposes, however, these problems are not essential. ${ }^{10}$

The second component of the cut argument is Heisenberg's claim that the statistical element can only appear at the location of the cut. His reasoning here is quite simple: on the quantum mechanical side of the cut, we consider a wavefunction that evolves univocally given initial values and is thus determinate, and on the classical side of the cut the properties of the system can of course be treated without appeal to statistics and the description is equally determinate. Thus, it must be at the place of the cut that the statistical element enters into our consideration of such a system.

Heisenberg does not provide an explicit conclusion based on these two elements of the cut argument in his 1935, nor indeed in any of his presentations of this argument. Instead, both in his Vienna lecture and in his 1935 draft (as we shall see) Heisenberg simply illustrates the point with his thought experiment from the letter to Einstein in 1927. We interpret the argument to be as follows: in order to be rid of the statistical elements and thereby provide a deterministic completion of quantum mechanics, one must

\footnotetext{
${ }^{10} \mathrm{~A}$ rigorous version of the arbitrary placement of the cut can be proved, along the lines of von Neumann's discussion in chapter VI of his (1932), or more generally, apply standard decoherence arguments.
} 
introduce hidden variables to describe the system's evolution across the cut. Since the position of the cut is arbitrary, one can imagine a scenario in which the cut is removed to some point beyond the measuring apparatus. However, the situation at the original placement of the cut is now overdetermined, since the quantum mechanical description is already determinate at that place. The further predictions that can be made on the basis of the hidden variables will in general contradict those of quantum mechanics. Thus, no such completion of the theory is possible. We shall now see in detail how Heisenberg develops this argument against hidden variables.

\subsection{Hidden variables}

In the second section of the EPR response, Heisenberg segues from the cut argument to a specific consideration of hidden-variables theories. The section opens with the following statements, which deserve special attention:

We shall thus investigate the assumption that the physical systems about which quantum mechanics makes statistical statements are bearers of hitherto unknown physical properties, that determine unambiguously the behaviour hitherto known only statistically. For example, let the nucleus of the radium atom possess, apart from the properties that are fixed by the knowledge of its stationary state, still other hitherto unknown properties whose knowledge makes possible an exact prediction of when the atomic nucleus will emit an $\alpha$-particle. And we assume specifically: this statement should hold independently of the means of observation that are used to detect the $\alpha$-particles.

One might at first be tempted to drop this last special assumption, and take into account that the radioactive emission can also depend on the properties of the means of observation.

Note that Heisenberg recognises as a separate assumption that hidden variables are to be considered independently of the means of observation. In other words, Heisenberg here appears to make for the first time a clear distinction between what we now call contextuality and non-contextuality in hidden-variables theories - a subtle point usually attributed to Bell some 30 years later.

Having made this distinction, Heisenberg first proceeds to dismiss as irrelevant the case of contextual hidden variables. His argument is based again on the movability of the cut: if the cut is placed well within what we would normally consider the classical side of the description (somewhere between the apparatus and the observer), then the assumption of contextuality is clearly unreasonable. From this Heisenberg concludes that it is unreasonable in general. This argument is, at best, hand-waving. ${ }^{11}$ The point to

\footnotetext{
${ }^{11}$ The mistake is obvious if one considers Bohm's theory, in which a measurement is modelled precisely as coupling an arbitrary quantum mechanical observable to an appropriate position observable, which can then be observed non-contextually.
} 
note, however, is that Heisenberg recognises the importance of the assumption of contextindependence to the degree that an argument for the appropriateness of this assumption is warranted.

After concluding that the context-independence of hidden variables is a necessary assumption for any such theory, Heisenberg argues that even with this assumption, hiddenvariables theories will come into contradiction with the outcomes otherwise predicted by quantum mechanics. Recall case (i) from above, in which the cut is placed between the atomic system $A$ and the first measuring device $B$. Under this arrangement, one has the freedom at $B$ to measure either a variable $q_{A}$ of system $A$ or the conjugate variable - say, $p_{A}$. Assuming the existence of hidden variables at the placement of the cut, these will fix in particular the value of $q_{A}$. Now consider the situation in which the cut has been removed beyond $B$, and suppose we wish to measure $p_{A}$. The quantum-mechanical predictions for $p_{A}$ will demand indeterminacy in the value of $q_{A}$, but this contradicts the existence of the hidden variables. Hence, no such completion of the theory can be made without violating the empirically well-confirmed predictions of quantum mechanics. ${ }^{12}$

One might paraphrase the last part of this argument as a restatement of what should be by now a familiar claim: the existence of hidden variables destroys interference - a claim much like the one found in Heisenberg's 1927 letter to Einstein. In fact, the thought experiment Heisenberg provides after giving the above general argument in his EPR draft is more or less identical to the thought experiment from his letter to Einstein in 1927, involving an $\alpha$-particle impinging on a diffraction grating. If the cut is placed at the screen, the existence of hidden variables at the grating will destroy the observed interference effects.

\subsection{Heisenberg and Hermann}

Before discussing the role of mutually exclusive observational contexts in the final section of his paper, Heisenberg summarises the argument against hidden variables he has just given, adding that its contents are essentially captured by the idea of the causal completeness of quantum mechanics as discussed in Grete Hermann's essay, 'Die naturphilosophischen Grundlagen der Quantenmechanik' (1935, 1996). Hermann, a former student of Emmy Noether and an enthusiastic member of Nelson's neo-Kantian school, went to Leipzig in 1934 for the express purpose of reconciling a neo-Kantian conception of causality with the new quantum mechanics. While in Leipzig, she had many discussions with Heisenberg and von Weizsäcker on questions concerning the philosophical interpretation of quantum mechanics. In the letter to Pauli accompanying his EPR draft, Heisenberg mentions Hermann's essay - possibly the first instance of a careful philosophical treatment of this new theory. He writes:

\footnotetext{
${ }^{12}$ Note that it is crucial for Heisenberg to consider both the movability of the cut and the possibility of measuring different observables. If only $q_{A}$ were to be measured, then no contradiction would ensue, since no further interference is required even in the quantum mechanical description.
} 
In particular I would like to deal with the question of the incompleteness of quantum theory. By the way, on this point there has recently appeared a halfphilosophical essay by Miss Grete Hermann, which has grown out of discussions in Leipzig, and which on the whole I find reasonable (but for the rationalist philosophical tendency). ${ }^{13}$

This is not exactly a ringing endorsement, although in the draft Heisenberg seems to agree with portions of her thinking. Exactly which portions, however, may prove difficult to reconstruct. One of Hermann's main claims in her essay is the consilience between causality and quantum mechanics, which she argues for by appeal to retroductive causation: although the cause for the appearance of a particular measurement result cannot be known prior to measurement, once the measurement has been made there is a univocal causal path that can be traced backwards to explain the given result. In this sense, according to Hermann, quantum theory is already causally complete, and thus in no need of supplementation with hidden variables, and Heisenberg appears to agree with this diagnosis.

But there is more to Hermann's essay that deserves mention here, as it relates directly to the larger context of Heisenberg's aim of arguing against the possibility of hidden variables. In her essay, Hermann devotes an entire section to a critique of von Neumann's (1932) impossibility proof for hidden variables - the section is titled 'Der Zirkel in Neumanns Beweis'. In this section, Hermann argues for a logical circularity in von Neumann, stating that the linearity assumption in the case of non-commuting observables is unjustified, and that in fact it characterises quantum mechanical states; thus von Neumann's proof becomes circular. Hermann's critique is similar in parts to that of Bell (1966), on which more will be said below.

Since Heisenberg evidently knew of Hermann's work, it is at least curious that he does not mention either this aspect of Hermann's work in his discussion of hidden variables, or in fact von Neumann's proof specifically (although he does cite von Neumann's book at the beginning of his draft, but not in connection to hidden variables). ${ }^{14}$ We shall return to this riddle in the next section.

\footnotetext{
${ }^{13}$ 'Inbesondere würde ich gern die Frage der Unvollständigkeit der Quantentheorie behandeln. Übrigens ist über diesen Punkt neulich eine halbphilosophische Abhandlung der Frl. Grete Hermann erschienen, die aus Diskussionen in Leipzig entstanden ist und die ich im Ganzen vernünftig finde (bis auf die philosophisch rationalistische Tendenz)' (Pauli 1985, p. 408).

${ }^{14}$ The question of why Heisenberg neglected to mention both von Neumann and Hermann in his discussion of hidden variables has been raised also by Léna Soler (2009) and Michael Seevinck (cf. his website, http://www.phys.uu.nl/igg/seevinck/, for a translation of 'Der Zirkel in Neumanns Beweis' and other work on Hermann). Both Soler and Seevinck consider various aspects of Hermann's work more fully. See in particular also Soler's introduction and commentary in Hermann (1996).
} 


\section{Other 'no-go' arguments}

We have identified three aspects of Heisenberg's approach to hidden variables in various writings between 1927 and 1935, namely the 'no-go' argument against hidden-variables theories, the treatment of interference and the cut argument. We believe that in considering specifically his 1935 draft and comparing it to his statements in earlier works, the relationships between these three aspects of his thinking become clearer. Specifically, we see how Heisenberg's account of interference shows that hidden variables are incompatible with interference phenomena; these considerations are then incorporated into a wider argument based on the movability of the cut, an argument he maintained well beyond 1935 .

We now turn to other 'no-go' arguments against hidden-variables theories current at the time - specifically, von Neumann's proof and various related arguments used in particular by Pauli and Schrödinger, partly in print but mainly in correspondence. While we do not give a comprehensive assessment of Schrödinger's and Pauli's views on the subject, our discussion of these lesser-known arguments suggests that the core of von Neumann's result was more widely appreciated and understood than is the folk belief. In our next and final section, we shall then attempt to characterise Heisenberg's approach to hidden variables as compared to these other approaches.

\subsection{Von Neumann's proof}

Von Neumann's impossibility theorem (1932, pp. 305-324 of the English translation) is a corollary of a wider theorem aimed at characterising possible ensembles of quantum mechanical systems. Von Neumann treats ensembles in terms of the associated expectation functionals on the observables. These expectation functionals are assumed to be positive, normalised and linear. Von Neumann states explicitly that linearity is operationally motivated only for commuting observables, and he states the general linearity condition as a separate assumption (pp. 308-309). On this basis, he then proves that expectation functionals are in one-to-one correspondence with quantum mechanical density operators.

Von Neumann then investigates homogeneous ensembles and dispersion-free ensembles. The former turn out to correspond to quantum mechanical vector states, which are not dispersion-free for all observables. But then it follows that there are no dispersion-free ensembles. This result is his impossibility proof. As a theorem, it is of course correct, but does it actually rule out hidden-variables theories?

The first criticism of von Neumann's assumption to have made a mark appears to have been J. S. Bell's in his celebrated 1966 paper. Bell pointed out not only that linearity is not operationally motivated, but that it is an unreasonable requirement to make on the hypothetical dispersion-free states. Indeed, for such states, it would imply that sums of eigenvalues of two non-commuting operators $A$ and $B$ should be eigenvalues of the sum $A+B$. However, this is impossible in general, and trivially so.

To see why this is the case, take the following example (Bell 1966). Consider the 
operators $\sigma_{x}, \sigma_{y}$ and $\sigma_{x}+\sigma_{y}$. For a linear, dispersion-free state $\lambda$,

$$
\left\langle\sigma_{x}+\sigma_{y}\right\rangle_{\lambda}=\left\langle\sigma_{x}\right\rangle_{\lambda}+\left\langle\sigma_{y}\right\rangle_{\lambda}
$$

where all terms need to be eigenvalues of the given observables. But the left-hand side takes the possible values $\pm \sqrt{2}$, while the right-hand side takes the possible values $-2,0,+2$, so that (7) cannot be satisfied at all.

According to the folklore, von Neumann's reputation was such and his mathematical sophistication was so far beyond the capabilities of the average physicist, that everyone believed his theorem did rule out hidden-variables theories until Bell's criticism over 30 years later. (Note that this did not prevent Einstein, Podolsky and Rosen from developing their incompleteness argument in 1935, nor Bohm from developing his hidden-variables theory in 1952 !)

Thus far the folklore. We have seen already that as early as 1935 Grete Hermann had criticised in print von Neumann's general linearity assumption as being unmotivated (and essentially begging the question). We now wish to point out that, also in 1935, both Pauli and Schrödinger in correspondence (and Schrödinger in print!) presented arguments quite similar to Bell's, though not as criticisms of von Neumann, but rather as 'quick and dirty' arguments against the possibility of hidden variables.

\subsection{Schrödinger and Pauli}

We shall now see that other arguments establishing directly the corollary of von Neumann's theorem seem to have been in the air at the time of Heisenberg's draft. In the summer of 1935, Schrödinger engaged a number of physicists in correspondence on the EPR paradox, and arguments of this kind appear in three of these letters, as follows. ${ }^{15}$ Pauli gives one in a letter to Schrödinger of 9 July (Pauli 1985, pp. 419-422), written exactly one week after Heisenberg had sent his EPR draft to Pauli; and Schrödinger gives two such arguments in his correspondence with Einstein. ${ }^{16}$ (This correspondence is rather more famous for containing Einstein's opinion of the EPR paper and his own preferred version of the incompleteness argument, as well as for discussions related to Schrödinger's cat; see for instance Fine (1986)). Schrödinger also presented versions of these two arguments in print, respectively in his well-known paper on entanglement (1935a) and his celebrated 'cat' paper (1935b). We suggest that the significance of these arguments has not yet been appreciated.

Here is Pauli's argument. He first states (Pauli 1985, p. 421, entire passage emphasised in the original):

\footnotetext{
${ }^{15}$ This paper is part of a wider book-size project based around the translation and commentary of this correspondence and other materials relating to the historical debate on the EPR paradox.

${ }^{16}$ Pauli to Schrödinger, 9 July 1935, Schrödinger to Einstein, 19 August 1935 and 4 October 1935, Archive for the History of Quantum Physics, microfilm 92, section 2 (all in German).
} 
[I]f one subdivides the quantum mechanical ensembles in subensembles and in the latter simultaneously assigns numerical values, with greater accuracy than the uncertainty relation allows, to quantities A, B belonging to non-commuting operators, then one comes into conflict with statistical claims of quantum mechanics about the total ensemble. ${ }^{17}$

He then presents the argument in detail for the following case. Take a pure ensemble with given momentum, and imagine dividing it into subensembles according to different values of position. (Note that in order to reproduce the quantum mechanical predictions, one will need these values of position to be distributed uniformly in the ensemble.) In this way it is possible to reproduce the separate expectation values of functions of $P$ and $Q$, and (thus) also of a sum $F=F_{1}(P)+F_{2}(Q)$. But it is not possible in general to reproduce the distribution for the values of $F$, thus also it is not possible in general to reproduce the quantum mechanical dispersion of $F$. As specific examples in which the distribution of values in the mixture of hidden states is different from that given by the pure quantum mechanical state, Pauli uses the energy of the harmonic oscillator, $F=P^{2}+\omega^{2} Q^{2}$, and the angular momentum, $F=P_{x} Q_{y}-P_{y} Q_{x}$, for both of which the distribution of values would be continuous in the mixture of hidden states, and discrete in the pure quantum state.

Pauli's argument could be said to be less forceful than Bell's, because the contradiction derived by Bell is at the individual level, while Pauli's is merely at the ensemble level (as he states, a contradiction with statistical claims of quantum mechanics). Still, Pauli's argument is perfectly valid. But that is not the end of the story.

Indeed, Schrödinger developed the argument further in his letter of 19 August to Einstein, deriving a contradiction already at the individual level. The argument was then repeated in his paper on entanglement, in the following passage (Schrödinger 1935a, pp. $560-561$, notation adapted):

One might presume [...] that the answer prepared for the variable $F(Q, P)$ is simply $F(q, p)$, if $q$ and $p$ are those prepared for $Q$ and $P$ respectively. But this is not so. For consider, e.g., the series of observables

$$
F(Q, P, b)=\frac{1}{b} P^{2}+b Q^{2}
$$

where $b$ is to be a positive $c$-number parameter. With every value for $b$ we are confronted with a new observable, to which an answer must be pending. Moreover the answer must be, irrespective of $b$, an odd integral multiple of $\hbar$ (though not necessarily independent of $b$ ). This shows plainly that all these answers cannot conform to the results which would be obtained by inserting into the expression the same pair of $c$-numbers, $p$ and $q[\ldots]$.

\footnotetext{
${ }^{17}$ '[W]enn man die quantenmech[anischen] Kollektive in Teilkollektive zerlegt und in den letzteren mit größerer Genauigkeit zu nicht vertauschbaren Operatoren gehörenden Größen A, B simultan Zahlwerte zuordnet als die Unsicherheitsrelation zuläßt, so kommt man mit statistischen Aussagen der $\mathrm{Qu}$ [anten]mechanik über das Gesamtkollektiv in Konflikt.'
} 
The point here is not only that $F$ has to take certain discrete values for any allowable pair of values of $P$ and $Q$, but that for any fixed pair of such values, i.e. at the individual level, $F$ has to take one or other of these discrete values for all real, positive values of $b$ (i.e. the energy of an oscillator has to be quantised for all possible values of the mass). And this is indeed impossible. (For instance, think of different rational and irrational values of $b$.) Thus, Schrödinger shows that, at the individual level, the constraints on the hidden states are strictly incompatible with the requirement that observed values of quantum mechanical observables be eigenvalues of the corresponding operators, much as Bell did with his simple spin example. ${ }^{18}$

Schrödinger's argument here appears to have gone unnoticed. Fine (1986, chapters 3 and 5) mentions it briefly in his analysis of the Einstein-Schrödinger correspondence, but makes very little of it. In the paper on entanglement, it comes as a brief remark after Schrödinger has proved one of his main results, namely that the EPR state is maximally correlated, so that the EPR argument can be applied to infer the existence of values for all observables. Schrödinger is thus pointing out the puzzling fact that, on pain of contradiction, these values cannot satisfy the functional relationships between observables. ${ }^{19}$

The argument may be hard to digest on a first reading, however. In his reply to Schrödinger, ${ }^{20}$ even Einstein confessed he had not followed Schrödinger's argument. So, in the following letter of 4 October, Schrödinger apologised for having been so unclear, and rephrased the example. Specifically, Schrödinger takes angular momentum, and points out that, quantum mechanically, angular momentum takes quantised values with respect to an arbitrary axis, while if one breaks down an ensemble into hidden states, then (we emphasise: for each individual hidden state) the same quantised values will appear only if angular momentum is evaluated with respect to certain privileged axes. And Schrödinger comments: 'This is terribly trivial' ('Das ist furchtbar trivial'). ${ }^{21}$

We see that Pauli and Schrödinger thought that a contradiction much like von Neumann's was perfectly obvious, given some simple (and at least classically natural) constraints on the hidden states. Pauli's argument is stated in terms of a statistical contradiction, while Schrödinger's obtains at the individual level, much like Bell's.

But are the constraints considered by Pauli and Schrödinger the same as those con-

\footnotetext{
${ }^{18}$ Note that Bell's example is simpler partly because he uses a two-dimensional Hilbert space, while Pauli's and Schrödinger's arguments require infinite dimensions, indeed use position, momentum and functions thereof.

${ }^{19}$ The next sentence is also suggestive, since Schrödinger seems to consider seriously the possibility that these values might indeed exist without obeying the functional relationships, which is another (modern) sense in which hidden variables can be contextual: 'Our complete lack of insight into the relationship between the different answers in one system is all the more bewildering, since we have proved, on the other hand, that the one-to-one correspondence between the answers of the two systems necessarily extends to all pairs of observables whenever it holds for two of them' (1935a, p. 561).

${ }^{20}$ Einstein to Schrödinger, 4 September 1935, Archive for the History of Quantum Physics, microfilm 92, section 2 (in German).

${ }^{21}$ This version of the argument is then repeated in print in Schrödinger's 'cat' paper (Schrödinger 1935b).
} 
sidered by von Neumann, Hermann and Bell? It appears that the assumptions used by Pauli and Schrödinger are the following: that the hidden states are dispersion-free, and that they respect the functional relations between observables (or at least between suitably symmetrised ones) - as opposed to linearity and dispersion-freedom, as assumed by von Neumann. How do these relate to each other? The assumption about functional relationships trivially implies the assumption of linearity. But, conversely, linearity and dispersion-freedom together imply also the respect of functional relationships. Thus, Pauli and Schrödinger (whether or not they were explicitly aware of the fact) were using the same constraints as von Neumann to derive (much more directly) a contradiction between hidden-variables theories and quantum mechanics.

The proof of the converse implication is straightforward. What one needs to show is that for any (symmetrised) polynomial $f(P, Q)$ and for any linear and additive state $\lambda$,

$$
\langle f(P, Q)\rangle_{\lambda}=f\left(\langle P\rangle_{\lambda},\langle Q\rangle_{\lambda}\right) .
$$

The result then follows by extension to power series. But (8) can easily be demonstrated by induction on the degree $n$ of the polynomial. It suffices to show it for special polynomials of the form $f(P, Q) X+X f(P, Q)$, where $X=P$ or $X=Q$, and $f$ is a polynomial of degree $n-1$. And indeed, if $\lambda$ is linear and dispersion-free, we have

$$
\begin{aligned}
\langle f(P, Q) X+X f(P, Q)\rangle_{\lambda} & =\left\langle(f(P, Q)+X)^{2}-f(P, Q)^{2}-X^{2}\right\rangle_{\lambda}= \\
& =\left(\langle f(P, Q)\rangle_{\lambda}+\langle X\rangle_{\lambda}\right)^{2}-\langle f(P, Q)\rangle_{\lambda}^{2}-\langle X\rangle_{\lambda}^{2}= \\
& =\langle f(P, Q)\rangle_{\lambda}\langle X\rangle_{\lambda}+\langle X\rangle_{\lambda}\langle f(P, Q)\rangle_{\lambda}
\end{aligned}
$$

and the result follows. Pauli's and Schrödinger's arguments thus establish directly the corollary of von Neumann's theorem by pointing out that some classically natural algebraic constraints and the assumption of dispersion-freedom (jointly equivalent to von Neumann's assumptions) are incompatible with quantum mechanics.

This is not to say that Pauli and Schrödinger thought of these assumptions as being unreasonable, as Bell would later argue. Pauli certainly presents his version as a bona fide argument against the existence of hidden variables. And in the absence of an alternative picture of what hidden-variables theories should be, it might be hard to come to think of these assumptions as unreasonable (despite the fact that Pauli had just seen the distinction between contextual and non-contextual hidden-variables theories in Heisenberg's draft!). ${ }^{22}$ It is unclear how seriously Schrödinger took his own version of the argument, but he presents it at least as a puzzle for a hidden-variables theory, and as a very obvious point to boot.

\footnotetext{
${ }^{22}$ As a matter of fact, we are not aware of how Pauli reacted to Heisenberg's arguments in the draft.
} 


\section{Static and dynamical approaches to hidden variables}

On the strength of our analyses of the approaches by Heisenberg and by others to the question of hidden variables, we can now attempt to define what is characteristic of these approaches, and how they differ.

All the approaches we have considered argue for contradictions between the predictions of a hidden-variables theory and those of quantum mechanics, but the contradiction arises differently for Heisenberg than for the other authors we have mentioned.

Von Neumann's, Schrödinger's and Pauli's arguments all have in common that they consider algebraic constraints on possible value assignments at a single time, and find that these constraints are in conflict with the requirements of quantum mechanics. This is a familiar strategy from later discussions of (non-contextual) hidden variables. One could characterise these as static approches.

By contrast, Heisenberg's approach is dynamical: according to Heisenberg, the existence of hidden variables at one time will destroy interference effects at later times, and in this way engender a conflict with the quantum mechanical predictions. Heisenberg is also the only author at this time who (even if briefly) considers that hidden-variables theories might be contextual, in the sense of a joint dependence of measurement results on both the hidden variables and the measurement context. These two aspects may well be connected: dependence on the measurement context is a dynamical feature of hidden-variables theories, and its possibility may have been realised by Heisenberg precisely because he thought of hidden-variables theories in dynamical terms, in terms of how hidden variables could produce or fail to produce results of measurements, rather than in terms of how they might abstractly define values for all quantum mechanical observables. ${ }^{23}$

One might speculate further as to what Heisenberg thought of von Neumann's proof and of any other arguments he may have been aware of, and of how his approach differed from other approaches. Indeed, he was presumably aware at least of von Neumann's proof and Hermann's criticism of it, and it is to be expected that he would have heard Pauli's arguments against hidden variables directly (even if perhaps only in reaction to his draft).

Perhaps Heisenberg thought that his approach was sufficiently different from von Neumann's (and others) that he need not worry about Hermann's criticism. Indeed, it appears that for Heisenberg, the mere existence of hidden values for at least one observable other than the one being measured guarantees a contradiction with quantum mechanics, irrespective of any constraints placed on such values. Heisenberg may have thought that Hermann's criticism was perfectly justified, and that his own approach was both less trivial and more to the point. Hermann's criticism of von Neumann is to be read within the larger context of her paper, in which she herself argues against the possibility of hidden variables, be-

\footnotetext{
${ }^{23}$ Although, as we have mentioned, experimental contexts play an important role already in Born and Heisenberg's 1927 Solvay report, and although the topic of 'Bohrian' observational contexts appears in the final section of Heisenberg's 1935 draft, it is not clear whether in his mind there is any connection between such contexts and the above considerations of experimental context in hidden-variables theories.
} 
cause quantum mechanics is already causally complete. Perhaps Heisenberg's remarks on Hermann in his 1935 draft indicate a much broader agreement between his approach and hers.

We hope in the above to have clarified at least in part Heisenberg's position on hiddenvariables theories, and some of the relevant context. Clearly, this paper has only scratched the surface of how Schrödinger, for instance, thought about hidden variables, or about the relation between Hermann's thought and Heisenberg's. We hope to address some of these issues in future work.

\section{Acknowledgments}

We would like to thank Chris Timpson, Michiel Seevinck, Kristian Camilleri and Léna Soler for discussions and references. We especially thank Don Howard for setting one of us (EC) onto Heisenberg's 1935 draft in the first place, and for suggesting to us the collaboration out of which this paper has grown. Finally, we thank the anonymous referee for helpful comments.

\section{References}

Bacciagaluppi, G. (2008), 'The statistical interpretation according to Born and Heisenberg', chapter 14 in C. Joas, C. Lehner and J. Renn (eds.), HQ-1: Conference on the History of Quantum Physics (Vols. I \& II), MPIWG Preprint Series 350 (Vol. II), pp. 269-288. Also under www.mpiwg-berlin.mpg.de/en/resources/preprints.html .

Bacciagaluppi, G., and Valentini, A. (2009), Quantum Theory at the Crossroads: Reconsidering the 1927 Solvay Conference. Cambridge: Cambridge University Press, forthcoming. On-line draft at arXiv:quant-ph/0609184v1 .

Bell, J. S. (1964), 'On the Einstein-Podolsky-Rosen Paradox', Physics 1, 195-200.

Bell, J. S. (1966), 'On the problem of hidden-variables in quantum mechanics', Reviews of Modern Physics 38, 447-452.

Beller, M. (1999), Quantum Dialogue: The Making of a Revolution. Chicago: University of Chicago Press.

Belousek, D. W. (1996), 'Einstein's 1927 unpublished hidden-variable theory: its background, context and significance', Studies in History and Philosophy of Modern Physics 27, 437-461. 
Bohm, D. (1952), 'A suggested interpretation of the quantum theory in terms of "hidden" variables', Physical Review 85, 166-179, 180-193.

Bohr, N. (1935), 'Can quantum-mechanical description of physical reality be considered complete?', Physical Review 48, 696-702.

Bohr, N. (1949), 'Discussion with Einstein on epistemological problems in atomic physics', in P. A. Schilpp (ed.), Albert Einstein: Philosopher-Scientist, The Library of Living Philosophers, vol. VII. La Salle: Open Court, pp. 201-241.

Born, M. (1926), 'Das Adiabatenprinzip in der Quantenmechanik', Zeitschrift für Physik 40, 167-192.

Born, M., and Heisenberg, W. (1928), 'Quantenmechanik', in Lorentz (1928). Translated in Bacciagaluppi and Valentini (2009), pp. 408-447.

de Broglie, L. (1928), 'La nouvelle dynamique des quanta', in Lorentz (1928). Translated in Bacciagaluppi and Valentini (2009), pp. 374-407.

Einstein, A., Podolsky, B., and Rosen, N. (1935), 'Can quantum-mechanical description of physical reality be considered complete?', Physical Review 47, 777-780.

Fine, A. (1986), The Shaky Game: Einstein, Realism and the Quantum Theory. Chicago: University of Chicago Press.

Gleason, A. (1957), 'Measures on the closed subspaces of a Hilbert space', Journal of Mathematics and Mechanics 6, 885-893.

Heisenberg, W. (1927), 'Über den anschaulichen Inhalt der quantentheoretischen Kinematik und Mechanik', Zeitschrift für Physik 43, 172-198.

Heisenberg, W. (1930), The Physical Principles of the Quantum Theory. Chicago: University of Chicago Press.

Heisenberg, W. (1949), Wandlungen in den Grundlagen der Naturwissenschaft. Zürich: S. Hirzel Verlag.

Heisenberg, W. (1958), Physics and Philosophy: The Revolution in Modern Science. New York: Harper and Brothers.

Heisenberg, W. (1985), 'Ist eine deterministische Ergänzung der Quantenmechanik möglich?', 
in Pauli (1985), pp. 407-418.

Hermann, G. (1935), 'Die naturphilosophischen Grundlagen der Quantenmechanik', $A b$ handlungen der Fries'schen Schule 6, 75-152.

Hermann, G. (1996), Les Fondements philosophiques de la mécanique quantique. Introduction and commentary by L. Soler; translated from the German by A. Schneel and L. Soler. Paris: Vrin.

Holland, P. R. (2005), 'What's wrong with Einstein's 1927 hidden-variable interpretation of quantum mechanics?', Foundations of Physics 35, 177-196.

Kochen, S., and Specker, E. (1964), 'The problem of hidden variables in quantum mechanics', Journal of Mathematics and Mechanics 17, 59-88.

Lorentz, H. A. (ed.) (1928), Electrons et photons: rapports et discussions du cinquième conseil de physique Solvay. Paris: Gauthier-Villars.

Popper, K (1959), 'The Propensity Interpretation of Probability', British Journal for the Philosophy of Science 10, 25-42.

von Neumann, J. (1932), Mathematische Grundlagen der Quantenmechanik. Berlin: SpringerVerlag. Translated as Mathematical Foundations of Quantum Mechanics, Princeton: Princeton University Press, 1955.

Pauli, W. (1985), Wissenschaftlicher Briefwechsel mit Bohr, Einstein, Heisenberg u.a., Band II: 1930-1939, K. v. Meyenn, A. Hermann and V. F. Weisskopf (eds.). Berlin and Heidelberg: Springer.

Schrödinger, E. (1935a), 'Discussion of Probability Relations between Separated Systems', Proceedings of the Cambridge Philosophical Society 31, 555-562.

Schrödinger, E. (1935b), 'Die gegenwärtige Situation in der Quantenmechanik', Die Naturwissenschaften 23, 807-812, 824-828, 844-849.

Soler, L. (2009), 'The Convergence of Transcendental Philosophy and Quantum Physics: Grete Henry-Hermann's 1935 Pioneering Proposal', in M. Bitbol, P. Kerszberg and J. Petitot (eds.), Constituting Objectivity: Transcendental Perspectives on Modern Physics, The Western Ontario Series in Philosophy of Science, Vol. IV. Springer, pp. 329-346. 